\title{
Adiabatic ionization potential of acetic acid and torsional dynamics of its cation
}

\section{Journal Article}

Author(s):

Zielke, Philipp; Forysinski, Piotr W.; Luckhaus, David; Signorell, Ruth (D)

Publication date:

2009-06-07

Permanent link:

https://doi.org/10.3929/ethz-b-000423660

Rights / license:

In Copyright - Non-Commercial Use Permitted

Originally published in:

The Journal of Chemical Physics 130(21), https://doi.org/10.1063/1.3149802 


\section{Adiabatic ionization potential of acetic acid and torsional dynamics of its cation}

Cite as: J. Chem. Phys. 130, 211101 (2009); https://doi.org/10.1063/1.3149802

Submitted: 19 March 2009. Accepted: 14 May 2009. Published Online: 01 June 2009

Philipp Zielke, Piotr W. Forysinski, David Luckhaus, and Ruth Signorell

\section{ARTICLES YOU MAY BE INTERESTED IN}

Photoionization of small sodium-doped acetic acid clusters

The Journal of Chemical Physics 134, 094314 (2011); https://doi.org/10.1063/1.3559464

IR plus vacuum ultraviolet spectroscopy of neutral and ionic organic acid molecules and clusters: Acetic acid

The Journal of Chemical Physics 125, 184308 (2006); https://doi.org/10.1063/1.2378626

Competitive fragmentation pathways of acetic acid dimer explored by synchrotron VUV photoionization mass spectrometry and electronic structure calculations

The Journal of Chemical Physics 137, 124308 (2012); https://doi.org/10.1063/1.4754273

\section{Lock-in Amplifiers up to $600 \mathrm{MHz}$}
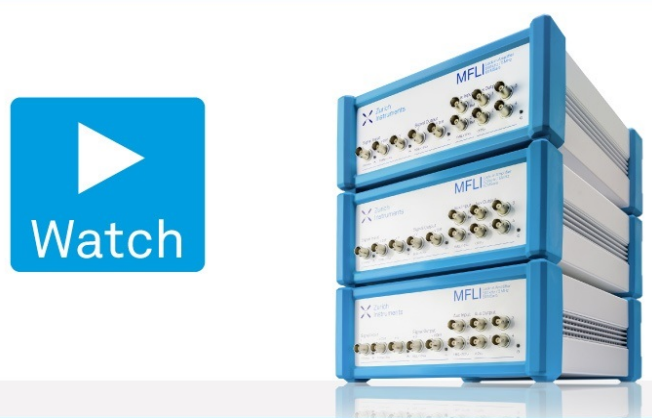

J. Chem. Phys. 130, 211101 (2009); https://doi.org/10.1063/1.3149802

130, 211101

(C) 2009 American Institute of Physics. 


\title{
Adiabatic ionization potential of acetic acid and torsional dynamics of its cation
}

\author{
Philipp Zielke, Piotr W. Forysinski, David Luckhaus, and Ruth Signorell ${ }^{\text {a) }}$ \\ Department of Chemistry, University of British Columbia, 2036 Main Mall, Vancouver V6T 1Z1, Canada
}

(Received 19 March 2009; accepted 14 May 2009; published online 1 June 2009)

\begin{abstract}
Pulsed-field-ionization zero-kinetic-energy photoelectron spectroscopy and supersonic cooling are used to investigate the $\mathrm{CH}_{3}$ torsional dynamics of the acetic acid cation and to determine an accurate value for the first adiabatic ionization potential of acetic acid $\left(\mathrm{IP}=85912 \pm 5 \mathrm{~cm}^{-1}\right)$, which has been the subject of debates for more than $40 \mathrm{yr}$. A doubling of the torsional barrier upon ionization is due to a significant shortening of the $\mathrm{C}-\mathrm{C}$ bond and reduces the tunneling efficiency by an order of magnitude. (C) 2009 American Institute of Physics. [DOI: 10.1063/1.3149802]
\end{abstract}

The interest in acetic acid $(\mathrm{AA})\left(\mathrm{CH}_{3} \mathrm{COOH}\right)$ and acetic acid cation $(\mathrm{AAC})\left(\mathrm{CH}_{3} \mathrm{COOH}^{+}\right)$in astrobiology arises from indications that $\mathrm{AA}$ is one of the building blocks of life. ${ }^{1}$ Glycine, e.g., is known to be formed from ammonia and AA. Since AA is found in the interstellar medium and in meteorites, it could have been brought to Earth by asteroids, comets, or meteorites. In unscreened regions of space, it is exposed to ultraviolet radiation, which in the extreme ultraviolet (XUV) region leads to ionization and fragmentation. While the spectroscopic properties of carboxylic acids have been investigated extensively, ${ }^{2-5}$ studies on the spectroscopy and photochemistry of their cations are comparatively limited $^{6-10}$ in spite of their essential role in understanding astrochemistry and thus the origin of life. The value of the first adiabatic ionization potential (IP) of AA has been debated for more than $40 \mathrm{yr}$. Reported values scatter between 10.35 and $10.70 \mathrm{eV}$, i.e., over a range of more than $2500 \mathrm{~cm}^{-1}$ (see Refs. 6, 8, and 10, and references therein). Other important issues with implications for the reaction kinetics of AAC concern the dynamics of its low frequency modes, especially the $\mathrm{CH}_{3}$ torsion with its unknown potential barrier $(\mathrm{H})$ and tunneling efficiency (Fig. 1). In the present contribution we employ pulsed-field-ionization zero-kineticenergy (PFI-ZEKE) photoelectron spectroscopy (see Refs. 11-14 and references therein) in combination with supersonic cooling to address these open questions.

The determination of the IP of AA and the torsional dynamics of AAC has turned out to be an experimental challenge. Photoelectron spectroscopy is the method of choice, but the resolution of spectrometers is often limited, while the extraordinary stability of the AA dimer further complicates the situation. The monomer is the dominant species only at high temperatures and low pressures, which can be realized in low pressure cells or effusive beams. Under such conditions, however, hot bands and thermally broadened rotational contours make the accurate determination of the IP and the torsional dynamics impossible. Figure 2(a) shows a simulation of the photoelectron spectrum of AA with the highest resolution yet reported. ${ }^{6}$ The bands are structureless and

${ }^{a)}$ Electronic mail: signorell@chem.ubc.ca. broad due to hot bands and broad rotational structure. No torsional structure is observed and the position of the IP remains unclear.

To tackle this problem, we combine a custom-built PFI-ZEKE photoelectron spectrometer with a supersonic expansion of $1.7 \%$ AA seeded in helium at a total pressure of 1 bar. ${ }^{13-15}$ This approach allows us to measure the photoelectron spectra of cold AA monomers selectively, very sensitively, and with high resolution $\left(0.5 \mathrm{~cm}^{-1}\right)$. The selectivity in PFI-ZEKE spectroscopy is achieved by laser excitation of very high lying Rydberg states that converge to a rovibrational level of AAC, which are field ionized $(0.19 \mathrm{~V} / \mathrm{cm})$ after a time delay $(2 \mu \mathrm{s})$. The time delay, together with a weak discrimination field $(0.09 \mathrm{~V} / \mathrm{cm})$, ensures that prompt ions and electrons (from AA and its oligomers) leave the ionization region before the Rydberg states are ionized and their electrons are detected. The excitation is effected in a single step by a custom-built ns-XUV laser (resolution of $0.5 \mathrm{~cm}^{-1}$ ), which is based on resonant four-wave mixing in a rare gas beam. Supersonic cooling of the sample is a prerequisite to reduce the contributions from hot bands and to narrow the rotational structure. Cooling, however, leads to the predominant formation of dimers and larger oligomers with only traces of monomer left in the sample, as illustrated in the time of flight spectrum in Fig. 3. Note that the mass resolution is limited as our setup is optimized for sensitive electron detection (defocused laser beam and short flight tube). The arrow indicates the very small AAC monomer peak, while all other peaks are fragments of oligomers. With only about 100 ppm AA monomer in the supersonic beam, demands on the selectivity and sensitivity of the electron detection are very high.

The PFI-ZEKE photoelectron spectrum in the region of the first adiabatic IP is shown in Fig. 2(b) together with a calculated spectrum for a vibrational temperature of $100 \mathrm{~K}$ in trace C. Photoionization spectra (ion signal not shown here) of AA and its oligomers confirm that the ZEKE spectrum arises solely from AA because only AA shows a threshold in this region. The main band at $85912 \mathrm{~cm}^{-1}$ is the transition between the vibrational ground states of the neutral $(v=0)$ and the ion $\left(v^{+}=0\right)$ (Fig. 1). Our simulation of the rotational 


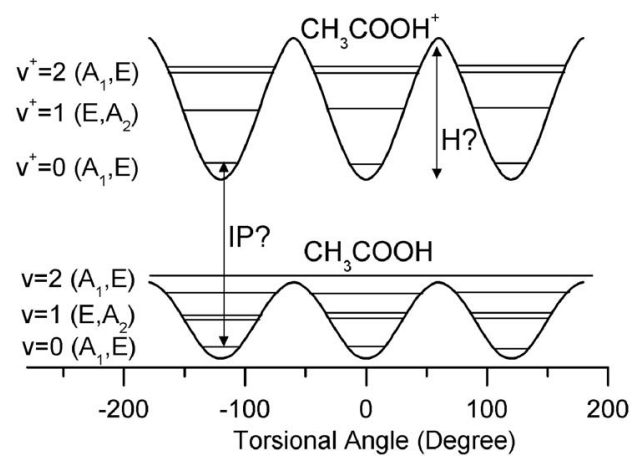

FIG. 1. Calculated potentials for the $\mathrm{CH}_{3}$ torsion $\left(\nu_{18}\right)$ of AA and AAC with corresponding energy levels (labels $v$ and $v^{+}$, respectively, and $\mathrm{C}_{3 v}$ tunneling symmetry labels in order of increasing energy).

structure shows that the width of this band is determined by the rotational structure with a rotational temperature of $10 \mathrm{~K}$. The $A, E$ torsional tunneling splitting as well as the rotational structure cannot be resolved with our laser. The calculated tunneling splitting is $0.35 \mathrm{~cm}^{-1}$ in AA and $0.06 \mathrm{~cm}^{-1}$ in AAC. The simulation of the rotational structure reveals a high average density of more than 100 transitions per $\mathrm{cm}^{-1}$. The bands on the high frequency side at 85937 and $85976 \mathrm{~cm}^{-1}$ are torsional transitions between states as labeled in Fig. 1. The second band system at $86269 \mathrm{~cm}^{-1}$ is the excitation of one quantum of the CCO deformation motion $\nu_{12}$ of AAC. This transition is also accompanied by $\mathrm{CH}_{3}$-torsional transitions on the high frequency side. The signal of this band system is about a factor of 3 less than for the first band system because the light intensity in this region drops by a factor of 3 due to the four-wave mixing efficiency. This band is scaled accordingly in Fig. 2.

The IP of AA, the torsional barrier height $\mathrm{H}$ of $\mathrm{AAC}$, and the corresponding tunneling splittings are derived from an analysis of the torsional structure observed in the ZEKE spectrum. We assume separability between rotational $|r\rangle$, $\mathrm{CH}_{3}$ torsional $|\tau\rangle$, vibrational $|v\rangle$, and electronic degrees of freedom with nonseparable interactions minimized by im-

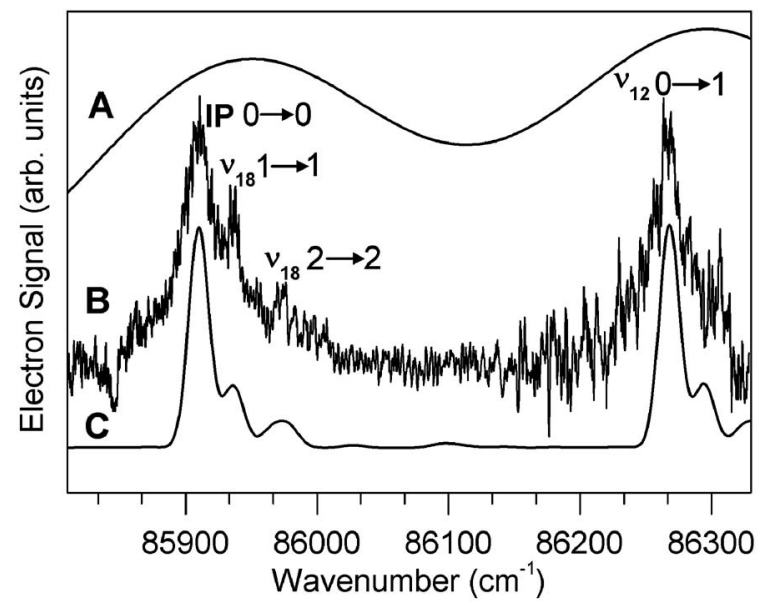

FIG. 2. (a) Calculated photoelectron spectrum of AA monomer at a temperature of $298 \mathrm{~K}$ and a resolution as reported in Ref. 6. (b) Experimental PFI-ZEKE photoelectron spectrum. (c) Calculated photoelectron spectrum at a vibrational temperature of $100 \mathrm{~K}$

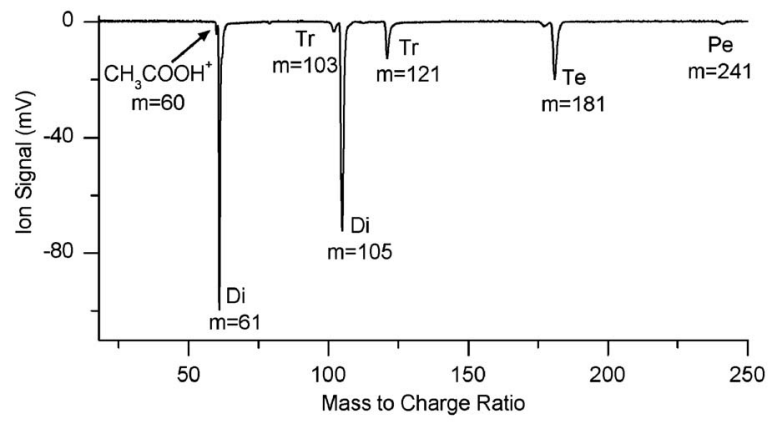

FIG. 3. Time of flight spectrum recorded at a laser photon energy of $85974 \mathrm{~cm}^{-1}$. The AAC monomer is labeled with an arrow. The spectrum is dominated by fragments of the dimer $(\mathrm{Di})$, the trimer $(\mathrm{Tr})$, the tetramer $(\mathrm{Te})$, and the pentamer $(\mathrm{Pe})$. Mass to charge ratio is given in $\mathrm{u} / e$.

posing orthogonality between infinitesimal vibrations, rotations, and torsions. Vibrations are treated as harmonic, except for the torsion, whose eigenfunctions are calculated explicitly in a discrete variable representation. ${ }^{16}$ Spectra were simulated within the Franck-Condon (FC) approximation for transition probabilities

$$
P\left(r^{+} \tau^{+} v^{+} \leftarrow r \tau v\right) \propto\left(\sum_{\ell} f_{\ell}\left(r, r^{+}\right)\right) \cdot\left|\left\langle\tau \mid \tau^{+}\right\rangle\right|^{2} \cdot\left|\left\langle v \mid v^{+}\right\rangle\right|^{2},
$$

where $(+)$ indicates ionic functions. The rotational contributions $f_{\ell}$, which depend on the photoelectron's orbital angular momentum $\ell$, are not known explicitly and therefore replaced with a phenomenological Gaussian band shape. The vibrational FC factors account for geometry change and Dushinsky rotation. ${ }^{17}$ The spectrum is given by the convolution of rotational, torsional, and vibrational factors. All equilibrium geometries, harmonic force fields, and torsional potentials were calculated within density functional theory $\left(\mathrm{B} 3 \mathrm{LYP} / 6-31+\mathrm{G}^{*}\right) .{ }^{18}$ At this level of theory the bare potential barriers (without zero point effects) along the minimum energy torsional path amount to 111 and $270 \mathrm{~cm}^{-1}$ for the neutral and the ion, respectively. In our calculations we employ these potential functions directly on the equidistant grid that defines the discrete variable representation for the solution of the torsional eigenvalue problem. ${ }^{16}$ The torsional potential of the neutral was scaled linearly by a factor of 1.53 to match the experimental barrier height of $170 \mathrm{~cm}^{-1}$. ${ }^{19}$ The analogous scaling factor for the torsional potential of the ion as well as the adiabatic IP and the torsional temperature were varied to match the ZEKE spectrum, resulting in an IP of $85912 \pm 5 \mathrm{~cm}^{-1}$, a torsional barrier of $H=316 \pm 10 \mathrm{~cm}^{-1}$, and a torsional temperature of $100 \mathrm{~K}$. In addition, the distance of $357 \pm 5 \mathrm{~cm}^{-1}$ between the two strongest transitions at 85912 and $86269 \mathrm{~cm}^{-1}$ is a direct measure of the CCO deformation mode of the AAC. The doubling of the torsional barrier height upon ionization hints at a significant shortening of the $\mathrm{C}-\mathrm{C}$ bond. B3LYP calculations predict a shortening of the $\mathrm{C}-\mathrm{C}$ bond by about $1 \mathrm{pm}$ largely independent of the basis set employed (1.6 pm for the PBE0 functional ${ }^{6}$ ), but the effect is more pronounced at the MP2/aug-cc-pVTZ level, which yields a bond length reduction by $2.1 \mathrm{pm}$. It may be instructive to note that even CCSD(T)/aug-cc-pVTZ (geometry optimized at the MP2 level) underestimates the 
torsional barrier by about 20\% (145 and $264 \mathrm{~cm}^{-1}$ for neutral and ion, respectively). The increase in the torsional barrier upon ionization drastically reduces the tunneling efficiency by almost one order of magnitude. The accompanying increase in the spacing of torsional energy levels produces corresponding changes in the partition function of AAC which will be most relevant at low temperatures.

In summary, we have shown that the combination of PFI-ZEKE photoelectron spectroscopy with supersonic cooling of an AA/helium mixture allows the determination of an accurate adiabatic IP of AA, thus ending a $40 \mathrm{yr}$ long debate. Supersonic cooling is essential to avoid the congestion caused by hot bands and broad rotational contours, while only a very sensitive and selective spectroscopic method can cope with the exceedingly small AA monomer concentrations in the supersonic expansion. Our experiments have enabled the analysis of the low frequency torsional dynamics of the cation, which will be important for statistical theories of the chemical reaction kinetics of AAC, especially at the low temperatures prevalent in space. Finally we would like to emphasize two more general aspects of this investigation. Our results illustrate the importance of detailed modeling for a reliable quantitative analysis of experimental photoelectron spectra, a point that has not always been appreciated as exemplified in occasionally exaggerated claims of accuracy. Second, this study demonstrates that with due attention to detail and optimization PFI-ZEKE photoelectron spectroscopy can achieve sufficiently high sensitivity to study molecules present only in trace amounts.

This work was supported by the Canada Foundation for Innovation, by the Natural Sciences and Engineering Re- search Council of Canada, and by the A. P. Sloan Foundation (R.S.). P.Z. acknowledges a postdoctoral fellowship from the German Research Foundation DFG.

${ }^{1}$ A. Brack, The Molecular Origins of Life (Cambridge University Press, Cambridge, England, 1998).

${ }^{2}$ E. M. S. Maçôas, L. Khriachtchev, M. Pettersson, R. Fausto, and M. Räsänen, J. Am. Chem. Soc. 125, 16188 (2003).

${ }^{3}$ P. Zielke and M. A. Suhm, Phys. Chem. Chem. Phys. 9, 4528 (2007).

${ }^{4}$ Y. H. Yoon, M. L. Hause, A. S. Case, and F. F. Crim, J. Chem. Phys. 128, 084305 (2008).

${ }^{5}$ Ö. Birer and M. Havenith, Annu. Rev. Phys. Chem. 60, 263 (2009).

${ }^{6}$ P. Limão-Vieira, A. Giuliani, J. Delwiche, R. Parafita, R. Mota, D. Duflot, J.-P. Flament, E. Drage, P. Cahillane, N. J. Mason, S. V. Hoffman, and M.-J. Hubin-Franskin, Chem. Phys. 324, 339 (2006).

${ }^{7}$ Y. J. Hu, H. B. Fu, and E. R. Bernstein, J. Chem. Phys. 125, 184308 (2006).

${ }^{8}$ S. Leach, M. Schwell, H.-W. Jochims, and H. Baumgärtel, Chem. Phys. 321, 171 (2006).

${ }^{9}$ L. Baptista, D. P. P. Andrade, A. B. Rocha, M. L. M. Rocco, H. M. Boechat-Roberty, E. F. da Silveira, E. C. da Silva, and G. Arbilla, J. Phys. Chem. A 112, 13382 (2008).

${ }^{10}$ D. J. Knowles and A. J. C. Nicholson, J. Chem. Phys. 60, 1180 (1974).

${ }^{11}$ K. Müller-Dethlefs, M. Sander, and E. W. Schlag, Chem. Phys. Lett. 112, 291 (1984).

${ }^{12}$ G. Reiser, W. Habenicht, and K. Müller-Dethlefs, J. Chem. Phys. 98, 8462 (1993).

${ }^{13}$ U. Hollenstein, F. Merkt, L. Meyer, R. Seiler, T. P. Softley, and S. Willitsch, Mol. Phys. 105, 1711 (2007).

${ }^{14}$ A. M. Schulenburg and F. Merkt, J. Chem. Phys. 130, 034308 (2009).

${ }^{15}$ P. W. Forysinski, P. Zielke, D. Luckhaus, and R. Signorell (2009) (unpublished).

${ }^{16}$ D. Luckhaus, J. Chem. Phys. 113, 1329 (2000).

${ }^{17}$ D. Luckhaus, Phys. Chem. Chem. Phys. 10, 6215 (2008).

${ }^{18}$ M. J. Frisch, G. W. Trucks, H. B. Schlegel et al., Gaussian03, Revision C.02, Gaussian, Inc., Wallingford, CT, 2004.

${ }^{19}$ V. Ilyushin, I. Kleiner, and F. J. Lovas, J. Phys. Chem. Ref. Data 37, 97 (2008). 